\title{
Thrombocytopenia as an initial sign of cirrhosis secondary to hepatic steatosis: a case report
}

Elvin Omar Videa-Irias ${ }^{1 *}$, Juan Jose Flores Perez ${ }^{2}$, Daniells Erazo ${ }^{3}$, David Alejandro Rodas Fino ${ }^{3}$, Scarlet Betzabel Rodas Gallardo ${ }^{4}$ and Michelle Elieth Trejo Cruz ${ }^{4}$

${ }^{1}$ Internal Medicine Department, School University Hospital, Tegucigalpa M.D.C., Honduras

${ }^{2}$ Internal Medicine Department, Hospital San Felipe, Tegucigalpa M.D.C., Honduras

${ }^{3}$ Faculty of Medical Sciences, National Autonomous University of Honduras, Honduras

${ }^{4}$ Student Faculty of Medical Sciences, National Autonomous University of Honduras, Honduras

\begin{abstract}
Cirrhosis is defined as the final stage of a chronic injury that modifies the normal hepatic architecture. Thrombocytopenia is a common complication in patients with chronic liver disease. We report the case of a 66 year-old female who presented to the emergency department with a cramp-type abdominal pain located in the right hypochondrium. Blood tests revealed a hemoglobin level of $11.7 \mathrm{~g} / \mathrm{dL}$, leukocyte count of $4,130 / \mathrm{mm}^{3}$ with neutrophil predominance, platelet count of 98,000 $/ \mathrm{mm}^{3}$ with prolonged coagulation times. An abdominal ultrasound reported the finding of cholelithiasis and liver with cirrhotic changes. We concluded that the thrombocytopenia was an initial sign of cirrhosis secondary to non-alcoholic hepatic steatosis with portal hypertension.
\end{abstract}

\section{Introduction}

Cirrhosis is defined as the final stage of a chronic injury in which structural abnormal regenerative nodules are surrounded by fibrous tissue that modifies the normal hepatic architecture [1-3].

NonalcoholicSteatohepatitisis defined as theexcessiveaccumulation of lipids in the liver, in abscence of secondary etiologies such as general or systemic disease, nutritional causes, genetic or metabolic, and drug induced hepatotoxicity. The nonalcoholic steatohepatitis (NASH) is one of the most aggressive clinical presentations of hepatic steatosis, in which the lipid infiltration is followed by intense necroinflammatory activity that can progress to cirrhosis and eventually liver failure and hepatocarcinoma $[4,5]$.

Thrombocytopenia is one of the most important clinical signs at the moment of diagnosis seen in 7 out of 10 patients who were diagnosed with this disease, which can also present spenomegaly and abnormalities in coagulation tests.

\section{Case report}

A 66 year old female patient comes to the emergency department presenting cholic abdominal pain located in right hypocondrium treated with analgesics, past medical history report hypertension for about 26 years, obesity grade I diagnosed by the BMI of 32 for 10 years and hepatic steatosis for 9 years, with no history of hepatitis.

A blood test revealed a hemoglobin level of $11.7 \mathrm{~g} / \mathrm{dl}$, leukocytes of $4,130 \mathrm{~mm}^{3}$ with neutrophil predominance, platelets $98,000 \mathrm{~mm}^{3}$. After finding thrombocytopenia and for Honduras being an endemic area for arbovirus a serological profile is requested which reports as negative. Coagulation test revealed altered prothrombin and thromboplastin time; other laboratory findings report normal bilirubin and transaminases.
With the possibility that the cause of thrombocytopenia were due to cirrhosis imaging studies were requested, a total abdominal ultrasound and a portal vein Doppler reported liver size of $13 \mathrm{~cm}$ with heterogeneous pattern and irregular edges, the portal vein with a flow of $15-20 \mathrm{~cm}$ per second spleen enlarged with $13 \mathrm{~cm}$ length (Figure 1), the gallbladder with a $2.1 \times 1.4 \mathrm{~cm}$ litho (Figure 2), no free liquid was identified; given these findings suspicion of cirrhosis and calculous chronic cholecystitis is confirmed and patient is prepare for cholecystectomy previous platelet transfusion. Cholecystectomy surgery was perfomed where a nodular pattern liver was visualized (Figure 3); a biopsy that reported hepatic tissue with rounded nodules formation of fibrous connective tissue with acute and chronic inflammatory infiltrate, fatty macrovascular metamorphosis $30 \%$

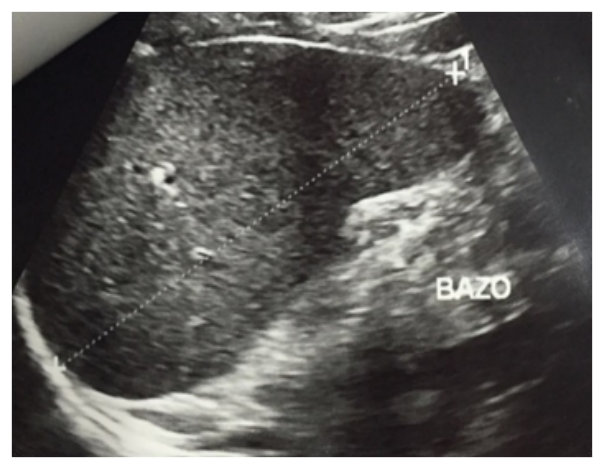

Figure 1. Spleen enlarged with $13 \mathrm{~cm}$ length.

Correspondence to: Dr. Elvin Omar Videa-Irias, Internal Medicine Department, School University Hospital, Tegucigalpa M.D.C., Honduras; E-mail: omar videa@yahoo.com

Received: October 22, 2016; Accepted: November 22, 2016; Published: November 26, 2016 
corresponding to Cirrhosis using the Brunt et al., grading and staging NASH it correspond to stage 3 which then develop to stage 4 (Figure 4).

Patient is discharged after surgery with good clinical evolution and a week later an upper digestive endoscopy is perform which rules out the presence of esophageal varices, to its antihypertensive management was added the therapy of antiportal hypertension which was propranolol of $40 \mathrm{mg}$ per day plus L-ornithine L-Aspartate once a day, the last platelets control show improvement with $135,000 \mathrm{~mm}^{3}$ and patient is regularly attending to follow-up medical evaluations (Table 1).

\section{Discusion}

Thrombocytopenia is often an incidental finding on routine laboratory testing, but can be indicative of important pathology. There are two main mechanisms for thrombocytopenia: (i) decreased production of platelets within the bone marrow and (ii) increased destruction or consumption of platelets in the periphery. The liver plays a key role in haematopoiesis and synthesis of coagulation

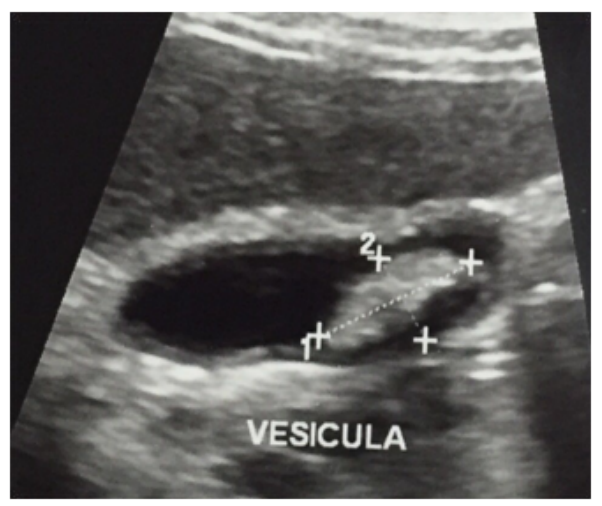

Figure 2. Ultrasound image of the gallbladder With a $2.1 \times 1.4 \mathrm{~cm}$ Litho. proteins, therefore liver disease is associated with a broad spectrum of haematological abnormalities.Thrombocytopenia is a common complication in patients with chronic liver disease and is seen in as many as $76 \%$ of cirrhotic patients. Traditionally, it was considered that thrombocytopenia in liver disease was exclusively due to increased pooling of platelets in an enlarged spleen, secondary to portal hypertension and extensive cirrhosis [6].

Liver disease was the most common cause for thrombocytopneia in outpatients, a common phenomenon caused by portal hypertension and congestive splenomegaly. The spleen can sequestrate around $90 \%$ of platelets in the bloodstream, even though it isn't rare to observe platelet serum levels below 50,000 cc, other prevalent causes included immune thrombocytopenic purpura (ITP) and primary bone marrow disorder. In inpatients, the causes of thrombocytopenia were more varied; liver disease accounted alongside sepsis/infection, anemia, folic acid deficiency, direct alcohol toxicity, hypersplenism, and bone marrow suppression $[1,6]$.

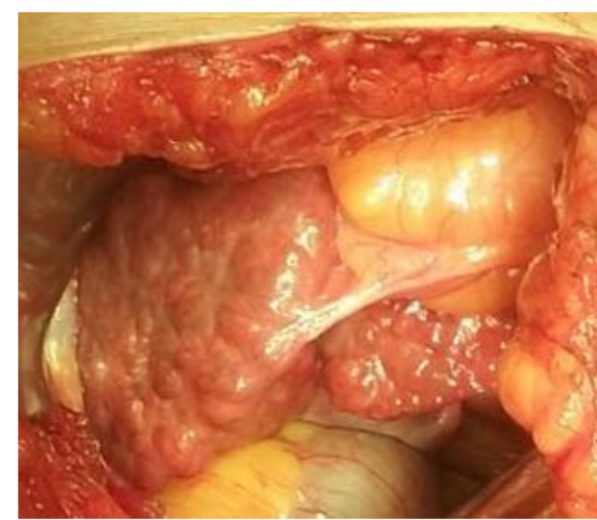

Figure 3. Nodular pattern liver.

Table 1. Laboratory Exams.

\begin{tabular}{|c|c|c|c|c|c|c|c|}
\hline & Day 1 & Day 7 & Day 23 & & Day 1 & Day 7 & Day 23 \\
\hline \multicolumn{4}{|l|}{ Blood Count } & \multicolumn{4}{|c|}{ Differential } \\
\hline Leukocytes & 4.13 & 7.80 & 4.09 & Neutrophils & 2.07 & 6.16 & 2.03 \\
\hline Erythrocytes & 4.51 & 4.20 & 4.18 & Neutrophils \% & 50.20 & 78.90 & 49.70 \\
\hline Hemoglobin & 12.30 & 11.50 & 11.70 & Lymphocytes & 1.66 & 1.21 & 1.60 \\
\hline Hematocrit & 36.70 & 33.90 & 34.80 & Lymphocytes \% & 40.2 & 15.50 & 39.1 \\
\hline Mean corpuscular volume & 81.40 & 80.70 & 83.30 & Monocytes & 0.38 & 0.41 & 0.44 \\
\hline $\mathrm{MCH}$ & 27.3 & 27.40 & 28.00 & Monocytes \% & 9.20 & 5.30 & 10.8 \\
\hline $\mathrm{MCHC}$ & 33.5 & 33.90 & 33.6 & Eosinophils & 0.01 & 0.00 & 0.01 \\
\hline Platelets & 98,00 & 108.00 & 135.00 & Eosinophils \% & 0.20 & 0.00 & 0.20 \\
\hline Meam platelet volume (MPV) & 11.40 & 10.80 & 12.00 & Basophiles & 0.01 & 0.02 & 0.01 \\
\hline \multicolumn{4}{|l|}{ Coagulation } & Basophiles \% & 0.20 & 0.30 & 0.20 \\
\hline Prothrombin time & 14.60 & & & \multicolumn{4}{|c|}{ Serology } \\
\hline INR & 1.08 & & & \multicolumn{2}{|l|}{ Dengue IgG, IgM } & \multicolumn{2}{|l|}{ Negative } \\
\hline TP Control & 12.00 & & & \multicolumn{2}{|l|}{ Chikungunya IgG, IgM } & \multicolumn{2}{|l|}{ Negative } \\
\hline Partial thromboplastin time & 27.80 & & & \multicolumn{2}{|l|}{ Zika } & \multicolumn{2}{|l|}{ Negative } \\
\hline Control TPT & 22.70 & & & \multicolumn{2}{|l|}{ Antibodies Helicobacter Pylori } & \multicolumn{2}{|l|}{ Negative } \\
\hline \multicolumn{2}{|l|}{ Chemistry } & & & & & & \\
\hline Total bilirubin & 0.40 & & & & & & \\
\hline Direct bilirubin & 0.18 & & & & & & \\
\hline Glucose & 99.14 & & & & & & \\
\hline Gamma Glutamil transpeptidase & 105.73 & & & & & & \\
\hline Aspartate amino transferase & 30.95 & & & & & & \\
\hline Alanine transaminase & 17.70 & & & & & & \\
\hline
\end{tabular}




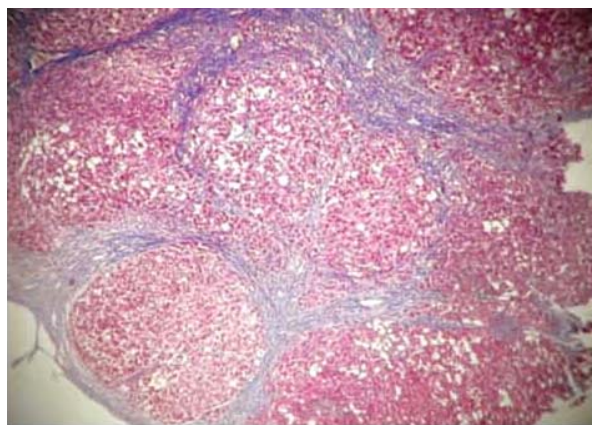

Figure 4. Thick bands of fibrosis delineating regenerative nodules, Cirrhosis (coloration trichrome Masson, 20X).

Fatty liver is the accumulation of triglycerides and other fats in the liver cells. In some patients, fatty liver may be accompanied by hepatic inflammation and liver cell death. Pathophysiologic mechanisms for fatty liver include (i) decreased mitochondrial fatty acid beta-oxidation (ii) increased endogenous fatty acid synthesis or enhanced delivery of fatty acids to the liver (iii) deficient incorporation or export of triglycerides as very low-density lipoprotein (VLDL) [7].

Four types of different histological variants can be observed in nonalcoholic steatohepatitis: type I showed steatosis alone, type II steatosis with lobular inflammation, type III steatosis with ballooning degeneration and type IV was similar to type III but also featured Mallory-Denk bodies and/or fibrosis [8].

About $10 \%$ of patients with NAFLD progress to NASH. Some 40 to $60 \%$ of patients with NASH-induced cirrhosis may develop a complication of cirrhosis including Hepatocelullar Carcinoma (HCC) after a period of 5 to 7 years of follow-up. 4 to $27 \%$ of NASH transform to HCC after the development of cirrhosis. In addition, most patients with HCC in the setting of NASH have also underlying diabetes (64\%), obesity (58\%) or other manifestations of the MS. Each year, hepatocellular carcinoma is diagnosed in more than half a million people worldwide. Liver cancer is the fifth most common cancer in men and the seventh in women. Hepatocellular carcinoma rarely occurs before the age of 40 years and reaches a peak at approximately 70 years of age $[9,10]$.

To date, several pharmaceutical agents have been used for the treatment of NASH; however, initial management must be focused on lifestyle modification and the reversal of conditions associated with NAFLD.

Lifestyle changes should include nutritional counselling with or without exercise. The pathophysiological basis for this approach is that weight reduction results in the loss of white adipose tissue, which decreases insulin resistance. Exercise can also improve muscular insulin sensitivity, which may improve the impact of insulin resistance on NAFLD. Several studies have shown that weight reduction with a calorie-restricted diet, with or without exercise, results in a significant biochemical improvement in overweight and obese adults and children. A few trials have also shown a significant reduction in ALT values in patients with biopsy proven NAFLD when they are put on calorierestricted diets with or without exercise Weight reduction is adopted, gradual loss not exceeding $1.6 \mathrm{~kg} /$ week should be emphasized through the combination of dietary restrictions and regular aerobic exercise regimen including at least $30 \mathrm{~min}$ of exercise three to five times per week [11].

Cirrhosis caused by nonalcoholic steatohepatitis presents platelet alterations, most commonly thrombocytopenia caused by splenomegaly secondary to portal hypertension, hematologic abnormalities such as anemia, macrocytosis and neutropenia, coagulation factor deficiency, disfribrinogenemia, hyperfibrinolysis, vitamin $\mathrm{K}$ deficiency secondary to malnutrition and malabsorption, and intravascular disseminated coagulation $[12,13]$.

\section{References}

1. Montoro M, Garcia Pagan J (2012)Gastroenterologia y Hepatologia Problemas Comunes de la Practica Clinica. 2nd ed. Ebook. Barcelona 867-890.

2. Schuppan D, Afdhal N (2008) Liver cirrhosis. The Lancet371: 838-851. [Crossref]

3. Pinzani, M., Rosselli, M. and Zuckermann, M. (2011). Liver cirrhosis. Best Practice \& Research Clinical Gastroenterology 25: 281-290. [Crossref]

4. CastroLS, Silva GP (2015) HÍGADO GRASO NO ALCOHÓLICO. Revista Médica Clínica Las Condes 26: 600-612.

5. Barraza EW, StalnikowitzKD (2016) ESTEATOSIS Y ESTEATOHEPATITIS NO ALCOHÓLICA. Revista de la Facultad de Medicina de la UNAM1-10.

6. Hancox S, Smith B (2013) Liver disease as a cause of thrombocytopenia. QJM 106 425-431.

7. Dawn, S. (2016). Fatty Liver: Overview, Pathophysiology, Etiology. Emedicine. medscape.com.

8. Hashimoto E, Tokushige K, Ludwig J (2014) Diagnosis and classification of nonalcoholic fatty liver disease and non-alcoholic steatohepatitis: Current concepts and remaining challenges. Hepatol Res 45: 20-28. [Crossref]

9. Rosmorduc O (2013) Relationship between hepatocellular carcinoma, metabolic syndrome and non-alcoholic fatty liver disease: Which clinical arguments? Annalesd'Endocrinologie, 74: 115-120. [Crossref]

10. El-Serag HB (2011) Hepatocellular Carcinoma. $N$ Engl J Med.365: 1118-1127. [Crossref]

11. Younossi (2016) Review Article: Current Management of NAFLD and NASH. Medscape.

12. Jimenez Cuadra E (2016) Articulo original. Complicaciones cirrosis hepatica Portalesmedicos.com.

13. Téllez-Ávila FI, Chávez-Tapia NC, Torre-Delgadillo A (2007) Trastornos de coagulación en el cirrótico. Revista de investigación clínica. 59: 153-160.

14. Tagle MA,PoggiLM, Ferrari NG, SiuHG,Aguinagac M,et al. (2008) Hallazgos clínicos, bioquímicos y de histología hepática en adultos peruanos con sobrepeso y obesos: primer estudio prospectivo nacional. Rev. gastroenterol. Perú 28: 323-331.

Copyright: (C2016 Videa-Irias EO. This is an open-access article distributed under the terms of the Creative Commons Attribution License, which permits unrestricted use, distribution, and reproduction in any medium, provided the original author and source are credited. 